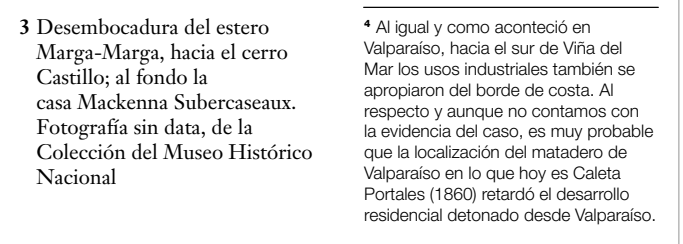

\begin{tabular}{c|c} 
ARQ & progresiva recualificación que para el caso de \\
52 & Valparaíso significaron el florecimiento de usos
\end{tabular} alternativos a los asociados al trasbordo de carga
y pasajeros. Provistos de instalaciones las más de y pasajeros. Provistos de instalaciones las más de
las veces provisorias, un ramillete de balnearios se ubicó en varios puntos de la línea de costa, antes dominadas por las faenas portuarias, el
quehacer de los pescadores $y$, más tarde, el febril quehacer de los pesc
trajín ferroviario trajín ferroviario.

seguramente demero a lo que imaginaríamos grupos sociales, hacia 1880 la lista de lo que un autor recientemente ha dado en llamar "sectores
de playa", era relativamente extensa: Cabritería, baños de La Caleta (la prolongación de Pacífico Álvarez (actual estación Bellhwista baños de la calle del Cabo (proximidades de la Plaza Echaurren), baños de los Almacenes Fiscales y playa San Mateo.

clientela en busca de baño y on to, una parte de los balnearios porteñ vio dificultada la obtención de su respectiv concesión, debido a la prolongación del ferrocarril que avanzó desde estación Barón hacia estación
Puerto. Devenida en una actividad disfuncion para el desarrollo del área, los balnearios debieron desplazarse hacia las nuevas localizacion balnearias adyacentes a Viña del Mar.

Viña del Mar antes de la ciudad jardín

La imagen urbana que Viña del Mar proyec la que el asentamiento exhibió durante su formación. Mientras en general su costa está

balnearios e infraestructuras de carácter público ace exactamente 100 años el borde estal tonado por arreas industriales que convivian co Tar, la costr acoǵn fábrizon de de Viña de y que vieron en el sector un adecuado $m$ de atributos: grandes paños, precios bajos conectividad al ferrocarril. De sur a norte, era nd Murphy (en lo que hoy es Castanza Lever el matadero de Viña del Mar (en la actualidad los edificios orientados desde Avenida Marin hacia el Casino de Viña del Mar), un astille emplazado en las cercanías del muelle Vergan (en lo que hoy son las playas que arrancan en 8

En claro contraste con el panorama industria dominante, el borde de costa también registró para dos balnearios: Miramar y Recreo. Provistos de playa y equipamientos especialmente acon-
dicionados para los bañistas (usualmente niños y ocasionalmente mujeres), Miramar y Recreo
se erigieron en lugares de exhibición para los se erigieron en lugares de exhibición para los
conspicuos visitantes que en verano buscaban diversión y ocio. Preferidos por los segmentos fueron incorporados al itinerarario social seguid por numerosas familias porteñas, santiaguin y también mendocinas. Del mismo modo,
ambos terminaron convirtiéndose en escenariós propiados para la localización adyacente amplios chalets y conocidas mansiones. Virtual epicentro de la vida cultural de las elites, Miramar también acogió el despliegue de nuevas
prácticas deportivas que se introducín en el

país. Tenis, ciclismo y fútbol fueron deportes practicados en la planicie abierta tras sucesiva ampliaciones. En este punto, cabe consignar que los atributos del balneario fueron suficientemente calibrados por el mercado del suelo. Lo anterior eniega presidencial en cerro Castillo y el cierre de la Maestranza Lever

\section{Reflexiones finales} Cido ferroviario Valparaíso-Santiago (1855) hubiese sido imposible urbanizar el litoral que arga-Maraiso de la desembocadura del estero los trabajos modifcoron sogras ind geografía de la costa, creando una potencia tranja de ocupación urbana ahí donde antes había un murallon de rocas agreste y escarpado. loteo de Viña del Mrobiliaria generada con a paseantes. Especialmente importante en dich proceso, Miramar es un ejemplo claro de cómo las estracturas balhearas pueden orientar el desarrollo de los habitantes. Precisamente, mientras sin presencia hubiese sido difícil romper la orientación mediterránea de Viña del Mar-dominada por uso industriales-, su dinamismo permitió convertir abierto, en un activo sufcientero mar como para redefinir el proyecto urbano de una ciudad completa a favor de su potencial balneario, tal y como se vería ratificado con la construccion

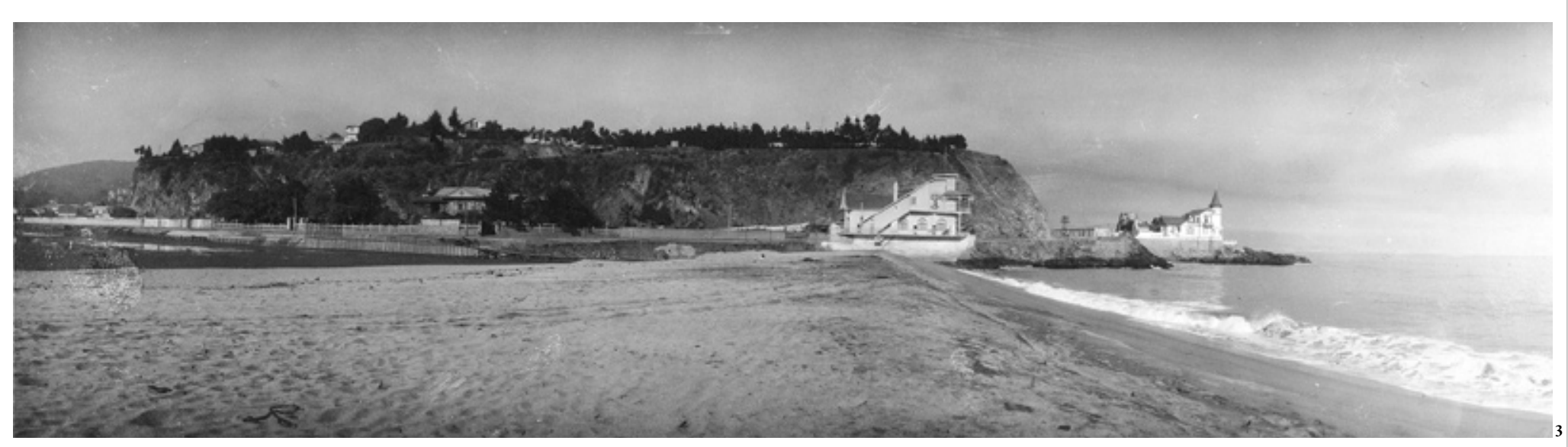

Un nuevo espacio de veraneo El Cap Ducal en la génesis de la modernidad'

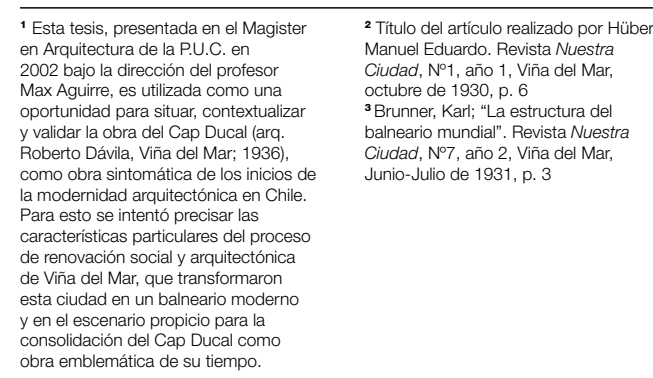

La sociedad d chilena de la primerer mitat d del siglo XX beralización de las costumbres, y la otra embarcada

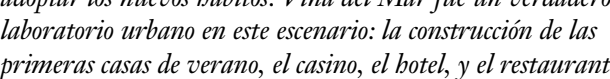

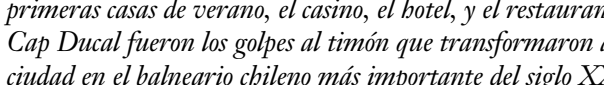

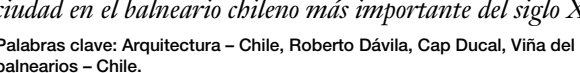

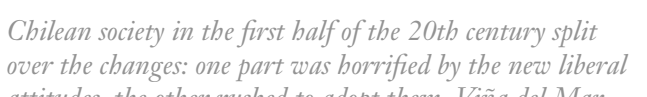

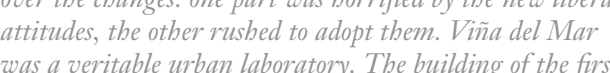

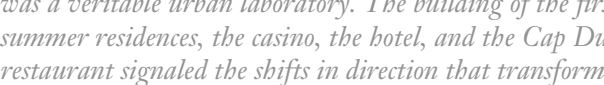

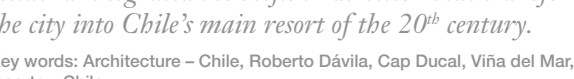
de Viña del Mar en un

esenciales, como ser la situación panorámica y mar, la distribución amplia de la población, la

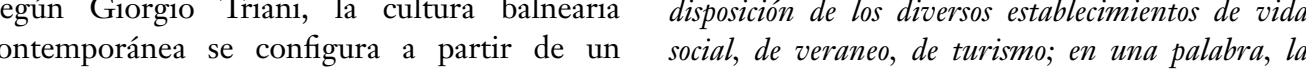

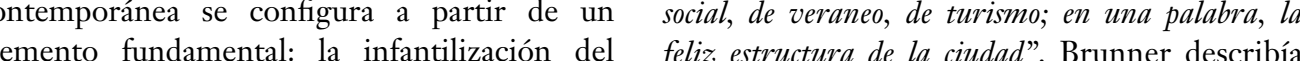

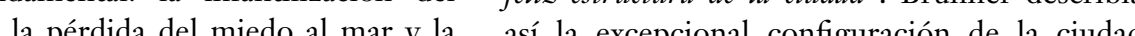
a modo de inmersión en la naturaleza, y por lo que realizaría la Municipalidad, "acaso la más tanto, en un placer.

(andad (órgano oficial de la octubre de 1930 y julio de 1931), publicaba en sus páginas tanto actividades sociales propias del verano (presentación de sus playas, piscinas, en general) como proyectos de arquitectura $\mathrm{e}$ infraestructura. De estos últimos se pueden nombrar obras tan importantes como el Casino
Municipal, el Hotel O'Higgins y el Palacio de Municipal, el Hotel O'Higgins y el Palacio de
los Presidentes en el cerro Castillo (todas ellas construidas simultáneamente). Además, se la valorización de la ciuddad, con títulos como: "El Veraneo en Viña", "Breve Semblanza del Balneario", o el siguiente escrito de Karl
Brunner: "Aquí, en Chile, tenemos un bahneario

progresista del país

alizar la corroborar a I municipio, principet tarea que venía realizando de 1906. La catástrofe significó un impuls decisivo en la instalación de la modernidad en Chile, y en este caso en particular marcó el se perfilaría como balneario.

En 1912 se creó la Sociedad Anónima Balneario de Viña del Mar. Ella adquirió los terrenos de Población Vergara, al borde del mar entre e

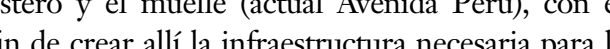
ansformación de la ciudad. Este lugar era el único que se encontraba vecino a la costa y al centro urbano, y por lo tanto, era el más apropiado par
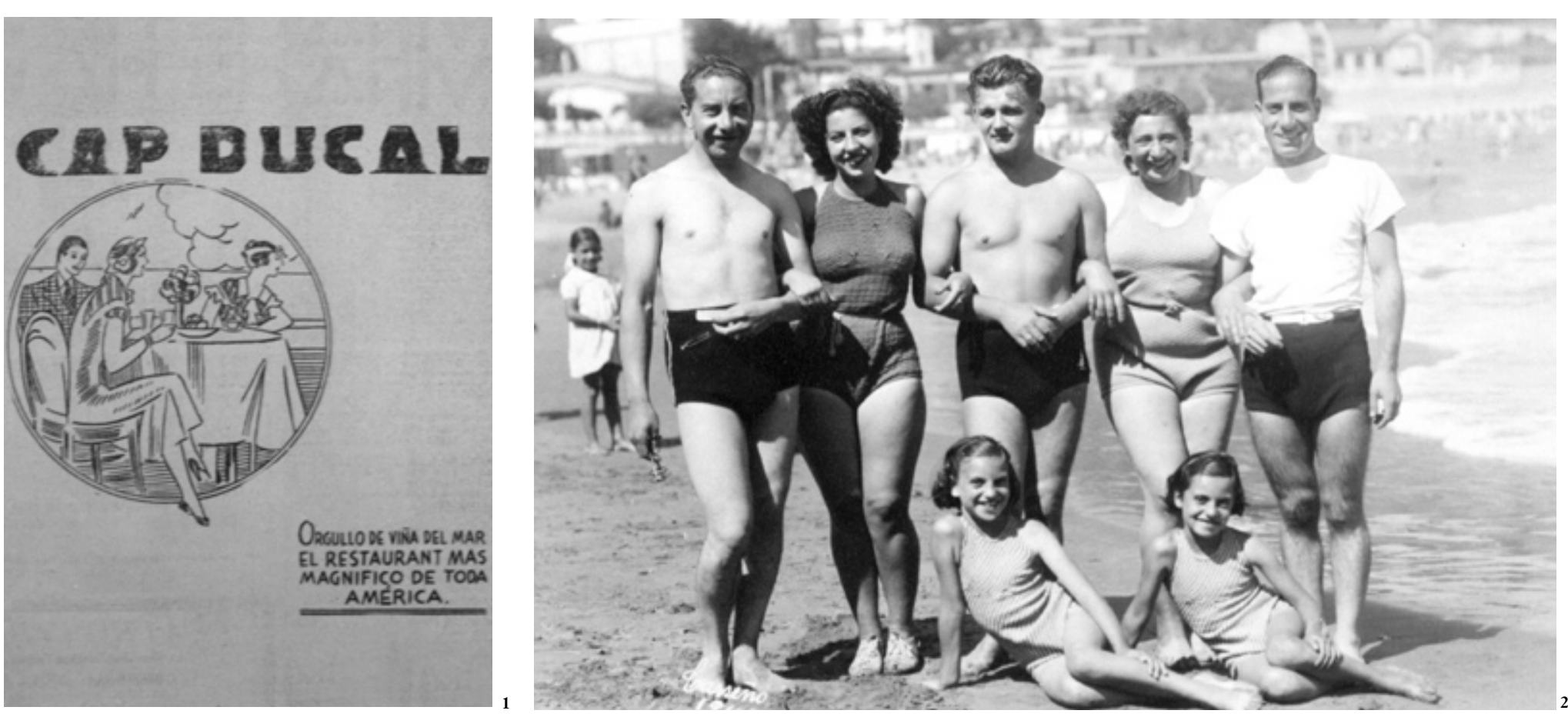

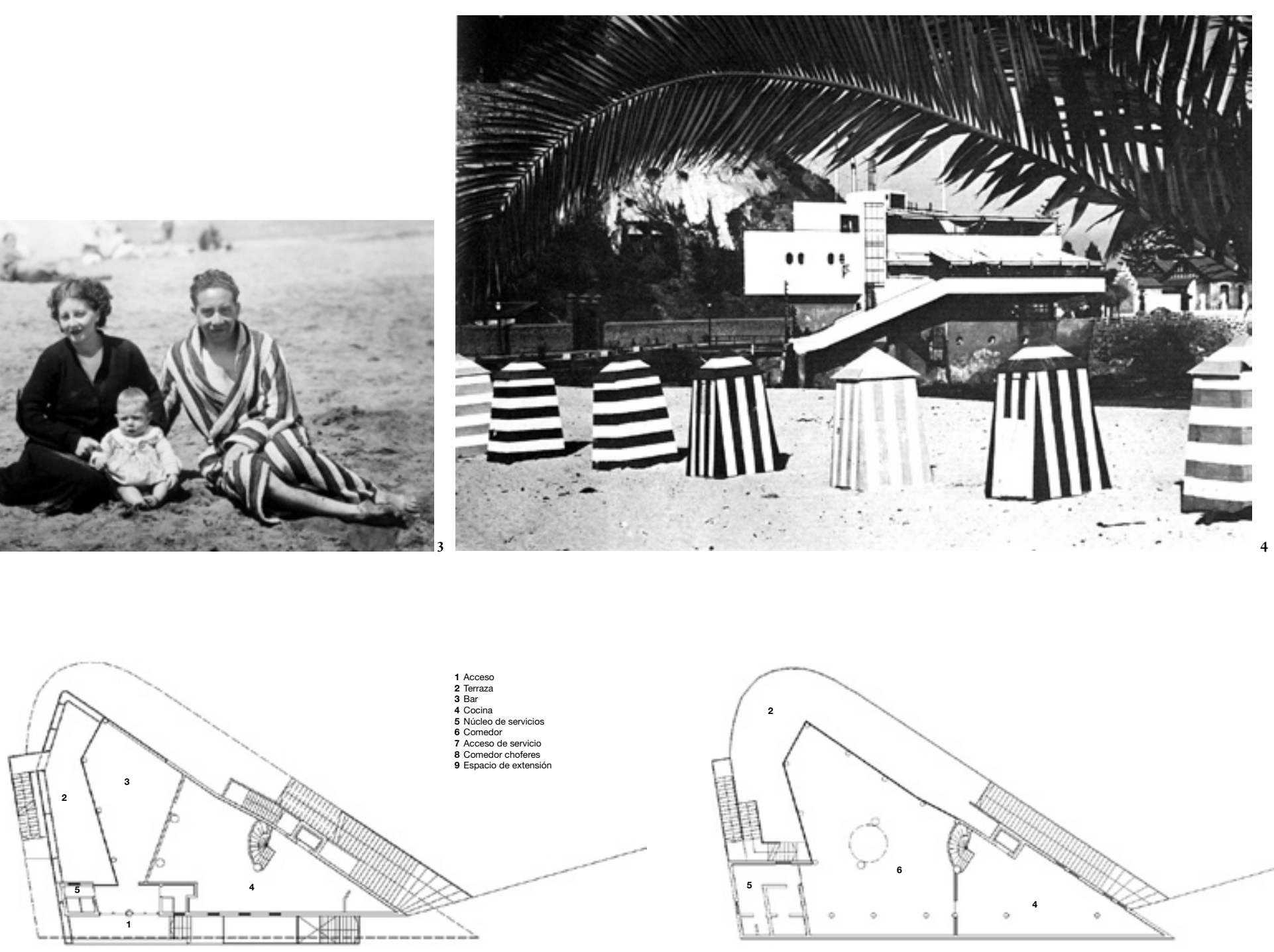

10 (II)

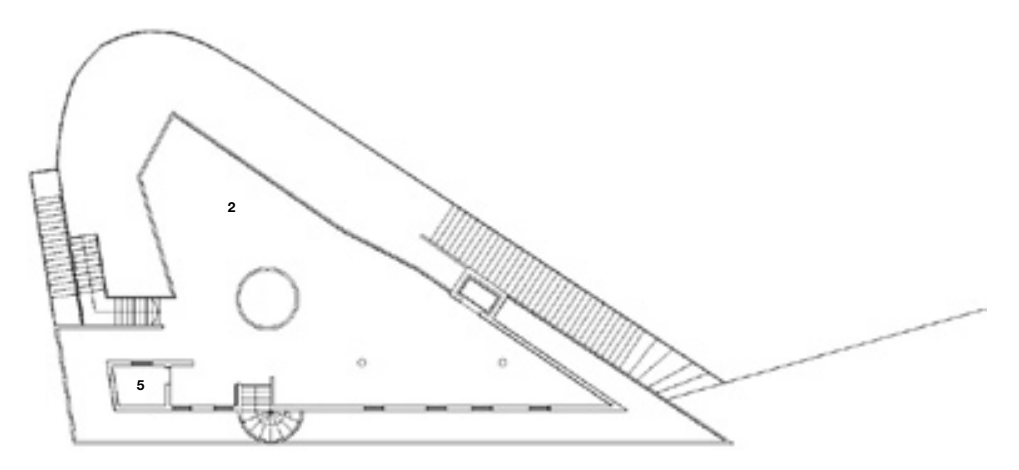

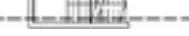

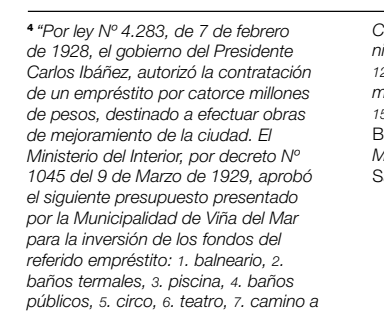

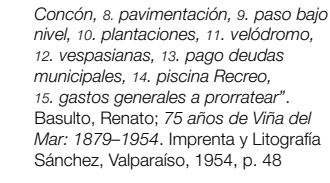

Pero es a partir de 1927, cuando Manuel Ossa asume como alcalde, cuando se realiza urbana. En 1928 se aprueba la ley No 4.283 de fomento al turismo y al progreso de la ciudad, en halnal se establece la creación de una junta profiscalizar y administrar las obras necesarias para estos fines'.

Dentro de ellas se encuentra el Casino de Viña, cuya inauguración en 1930 causó un gran pero que se transformó en un importante foco de atención turístico y social, complementario a las actividades del verano, que genero una fuerte entrada de dinero a la Municipalidad. O'Higinins en 1935, que albergrín a los Hotel ofreciendo las máximas comodidades

Otra obra de relevancia fue el Palacio de los Presidentes de Chile (inaugurado por Carlos Ibanez del Canpo el 19 de febrero de 1930 al reservar a los Presidentes un espacio de veraneo y descanso en la ciudad. Esta acción, política por cierto, avalaba la gestión de la junta

Dos conceptos básicos planteados por la la comprensión que las obras de arquitectura no sólo por su utilidad (o programa), sino que también por su representatividad, podrían otro lado lo dechandacionocín del estilo de vida de la sociedad chilena, que incorpora en sus rutinas la vida al aire libre, las vacaciones, el deporte y el encuentro social, en particular

A las tres obras descritas habría que agregar
la creación de las piscinas de 8 Norte $(1929)$ de Recreo (1930), así como la habilitación de diversos balnearios: Recreo (1924), Las Salinas (1929), Caleta Abarca y Cochoa (1935). Estas playa, el mar y el sol, convirtiendo a la ciudad de Viña en "expresión de la problemática y modiffcación del gusto que experimentaba nuestra sociedad"
llevándola a una cierta liberalización de sus llevándola
costumbres.

estente de esta ocupación aparece del Cap Ducal. En 1884 el doctor Teodoro Vo Schroeders estableció - con fines terapéuticoslos baños de Miraniar, que otorgaban espacio cada vez más populares baños de mar (que

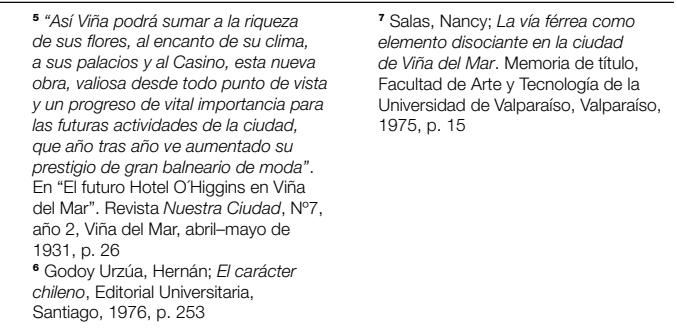

incidirían positivamente en la salud de quienes colución de diversos medios de transporte (como el tren y el automóvil) que facilitan lagresivamente la colonización de la costa y
las playas. Desde la Revolución Industrial el tren aparece artir de la creación de uaco hacia el hiterior, de moda del pais entero" perticular, y precisamente después de los años Roberto Dávila, Jorge Arteaga, y de Costablo El Cap Ducal sería uno de los espacio practicaban). Su ampliación en 1887 permitió specialmente la playa con baños calientes, hroeders, para motivar su uso dentro de la conservadora sociedad de la época.

Otro de los fictores a due inflý en como uno de los elementos claves en la primer momento, la expansión urbana de Viñ h ciudad con las zonas agrícolas de Olmué ceación de los mencionados baños de Miramar onstituyo el primer esfuerzo de apropiación de una estrín ferroviaria que uń los bĩos c entro urbano en 1885. Este proceso de ás elocuente en la llegada del tren desto Santiago en 1924. "La linea férrea constitityó par desde ese momento fue para chilemes y extranierque el sitio de atracción más interesante de la región... desd dia de la inauguracion del ferrocarril, se hizo el sitio

Es transforma en una ambios sociales que acompañan el surgimicto de las vacaciones y del veraneo. En este contexto nertará la obra del Cap Ducal.

\section{odernidad local} 政 construcción de la Avenida Marina en 1912 se Palacio Ducal, propiedad de Alberto Mackenna Mercedes Matte. Posteriormente, don Doming Tocornal Matte adquarió el salón de té y llamố Garafulic, para la construcción de un nuevo restaurante sobre los cimientos del antiguo balneario de Viña del Mar para dar lugar a la

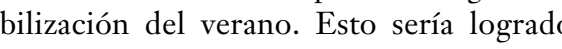
ares proposición arquitectónica 作

prontanta propone claramente una secuencia tal, en la relación entre interior núleo rígido hacia la calle, espacios de
no te se ar desarollados horizontalmente structura de pilares y ventanales, $y$ finalmente, espacios de extension tambien desarrollados sobre el mar.

espacial era reforzada por los rogramas que estos espacios contenían: un bord uro de servicios que independiza el restaurante

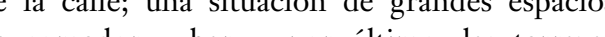
que se relacionan con la vista $y$ el mar. Toda orizontal, responde también a una condición de lagar donde la obra se abre completamente con uperficies de suelo y cielo que se proyectan hacia cerándose a la calle con un achada dura.

Esta operación de descomposición es realizada disminuyen la vertical a través de losas que atura abriéndose cada vez más a las terrazas, une se abalconan hacia el mar, hasta generar un Las operaciones analizadas no tendrían mayo valor, si es que los espacios no estuviese (escaleras), que configuran un sistema de ecorrido continuo, ascendente y periférico al $Y$ es que le da sentido a la obra.

de radical ina de recorrido del espectador es mplazamiento en el borde costero. Esto porque ecorrido circunvala la obra estableciendo su limite como una línea dinámica, relacionada con

Es así como la obra, a través de su propuesta quuitectónica, reacciona a la condición específica forma intenta responder a las nuevas exigencias del balneario moderno en que se transformaba la del siglo XX. ARa
$\underset{55}{\mathrm{ABQ}}$

rquitectónicos 